\title{
Hospital-Acquired Infections After Cardiac Surgery and Current Physician Practices: A Retrospective Cohort Study
}

\author{
Scott O’Keefe ${ }^{\mathrm{a}}$, Kenneth Williams ${ }^{\mathrm{a}}$, Jean-Francois Legare ${ }^{\mathrm{a}, \mathrm{b}}$
}

\begin{abstract}
Background: The management of hospital-acquired infections (HAIs) with respect to physician practices remains largely unexplored despite increasing efforts to standardize care. In the present study, we report findings from a 2-month audit of all patients that have undergone cardiac surgery at a large referral center in Atlantic Canada.
\end{abstract}

Methods: All patients who underwent cardiac surgical procedures during May and June 2013 at the Queen Elizabeth II Health Sciences Center in Halifax, Nova Scotia were identified. The prevalence of urinary tract infections (UTIs), pneumonia, leg harvest site infections, superficial sternal wound infections, deep sternal wound infections, and sepsis was examined to determine physician approaches in terms of verification rates (microbiology), time of diagnosis and duration of treatment. Continuous variables were compared using Student's $t$-test and categorical variables were analyzed using Fischer's exact test.

Results: A total of 185 consecutive patients underwent cardiac surgical procedures, of which 39 (21\%) developed at least one postoperative infection. The overall prevalence of infection types, from highest to lowest, was UTI (8\%), pneumonia (7\%), leg harvest site infection $(5 \%)$, superficial surgical site infection (4\%), and sepsis (2\%). There were no deep sternal wound infections. The overall in-hospital mortality rate was $3.8 \%$ with a median length of stay (LOS) of 8 days. The overall infection verification rate was 50\% (ranged from 100\% in sepsis to $10 \%$ in leg harvest site infections). In all cases, a full course of antibiotics was administered despite negative microbiology cultures or limited evidence of an actual infection.

Conclusions: HAIs are commonly treated without being verified and treatment is often not discontinued after negative cultures are received. Our findings highlight the fact that antibiotic treatment is not

Manuscript accepted for publication June 07, 2016

aDepartment of Surgery, Division of Cardiac Surgery, Dalhousie University, Halifax, NS, Canada

${ }^{\mathrm{b} C o r r e s p o n d i n g ~ A u t h o r: ~ J e a n-F r a n c o i s ~ L e g a r e, ~ M a r i t i m e ~ H e a r t ~ C e n t e r, ~ H a l i-~}$ fax Infirmary, Queen Elizabeth II Health Sciences Center, Dalhousie University, 2269-1796 Summer St, Halifax, NS B3H 3A7, Canada.

Email: legarej@me.com

doi: https://doi.org/10.14740/jocmr2637w always supported by evidence, and the effect of this could contribute to increased selective pressure for antimicrobial resistant bacteria.

Keywords: Hospital-acquired infection; Infection verification; Antimicrobial stewardship

\section{Introduction}

Hospital-acquired infections (HAIs) are a significant problem in Canadian health care, leading to higher mortality, longer hospitalization and increased use of health care resources [1, 2]. We have recently published observations on the prevalence of HAIs in adult post-cardiac surgery patients at one Canadian cardiac surgery center, showing an increased prevalence over an 18 -year period from $8 \%$ in 1995 to $20 \%$ in 2013 [3]. Furthermore, this occurred despite advances in infection control practices that have been implemented to allow the institution to receive national accreditation. It is also important to highlight the fact that we demonstrated that our observed rates of increased infection occurred independent of patient risk factors such as age, urgency or important co-morbidities.

While this trend in HAIs is alarming and has resulted in a series of initiatives to better address the problem. Of particular interest are previous studies that have shown that infections are commonly diagnosed with limited clinical criteria and often treated without lab confirmation [4]. Beyond this, antibiotic treatment is often continued after negative lab results are received [5]. Antimicrobial stewardship suggests that patients with unconfirmed infections in which antimicrobials are discontinued after 3 days have similar outcomes to those who receive full treatment [6]. Physician stewardship is believed to be a key component in reducing the prevalence of antimicrobial resistant bacteria, which involves the proper administration of antimicrobials. Early initiation of treatment and minimization of its duration has been shown to reduce the probability of antimicrobial resistance epidemics in hospitals [7].

Diagnosing HAIs in postoperative cardiac patients can often be difficult due to several causes other than infection that lead to inflammation, such as tissue damage and systemic inflammatory response syndrome (SIRS) [8]. Systemic anti- 
microbials administered before, during and after surgery may also contribute to lower blood culture yields, making confirmation of infection more difficult [9]. While these factors may cause problems in positively identifying infection, verification of infection is still a very important aspect of patient care as it allows physicians to prescribe the optimal antimicrobial as well as cease treatment if lab results are negative and/or clinical improvement occurs. In addition, community and hospitalacquired antimicrobial resistant bacteria are becoming a more prevalent global problem and measures to mitigate infection by these bacteria species need to be undertaken to avoid further complications in patients [10].

The practice of documenting, confirming or verifying HAIs has yet to be adequately studied or reviewed. In the present study, we extended our previously published findings to conduct a detailed chart review from a 2-month sample period of patients that underwent cardiac surgery at tertiary cardiac care center [3]. Patients acquiring at least one type of infection postoperatively were identified and further analyzed to better assess current physician practices.

\section{Methods}

\section{Setting and patients}

All consecutive patients who underwent cardiac surgical procedures during May and June 2013 at the Queen Elizabeth II Health Sciences Center (QEII HSC) in Halifax, Canada were identified. The period of time chosen for this audit represents $20 \%$ of the annual volume of cardiac surgery performed in Halifax. The QEII HSC is the only cardiac surgical center in the province of Nova Scotia and serves a population of almost 1 million. The Maritime Heart Center (MHC) registry is a prospective registry that has been used since 1995 and provides data on all cardiac surgical patients undergoing procedures at the QEII HSC. All patients were identified using this registry. What is unique about this study compared to previous work by our group is that the registry data were supplemented using the Horizon Patient Folder (HPF) database. HPF represents the PDF's version of all the actual medical records of each individual patient treated at the QEII HSC.

\section{Standard procedures related to HAIs}

Institutional established standard operating procedures have been in place and continue to follow national guidelines at the QEII HSC. Standard practice includes the use of prophylactic antimicrobials administration prior to skin incision (first generation cephalosporin or vancomycin in penicillin-allergic patients). Skin preparation has remained largely surgeon-dependent and included a combination of iodine and alcohol or a chlorhexidine-based solution. Placement of central catheters prior to surgery was done under strict sterile conditions in the operating room by anesthesiologists. Urinary catheters were also placed under sterile conditions in the operating room and were removed as soon as patients began to ambulate. Standard pneumonia prevention protocols were in place during the study period and included mouth care, subglottic secretion drainage and semi-recumbent position.

\section{Study design}

Patients were classified as having a postoperative infection if at least one of the following three criteria were met: a wound opening that involved excision of tissue; positive culture from respiratory secretions or pleural fluid, wound, blood, or urine; or antibiotic treatment based on clinical suspicion. The Society of Thoracic Surgeons (STS) data definitions were used to define how an infection was captured by the MHC registry and represent a well-known benchmark in cardiac surgical literature (sts.org).

We selected six types of infections based on recently published work by our group and due to their high relevance for our study population [3]. These included: 1) urinary tract infection (UTI), 2) pneumonia, 3) harvest site infections, 4) superficial sternal site infection (sSSI), 5) sepsis, and 6) deep sternal site infection (dSSI).

Clinical characteristics of patients that were identified as having an infection or not were examined univariately. Data captured also included: type of infection, verification through laboratory microbiology cultures, date of bacteria detection, type of antimicrobials used, duration of antimicrobial treatment, whether antimicrobials were discontinued after suspected infection was confirmed negative, and the type of bacteria identified. The outcomes of interest included mortality and length of stay (LOS).

Continuous variables were compared using Student's $t$ test and categorical variables were analyzed using Fischer's exact test. The Prism 6 (Graph Pad PRISM6 ${ }^{\circledR}$ ) statistical software package was used to complete all statistical analyses.

\section{Ethics approval and consent to participate}

The study was completed with full approval from the institutional (Capital District Health Authority) Research Ethics Board (REB). Given the retrospective nature of the work, a waiver of consent was granted by the REB.

\section{Results}

\section{Patient population}

During the time period of May and June 2013, 185 consecutive patients underwent cardiac surgical procedures requiring a heart-lung machine at the QEII HSC. Most patients were male $(82 \%)$, had an average age of $64.4 \pm 12.6$ years, underwent isolated coronary artery bypass graft $(\mathrm{CABG})$ procedures $(52 \%)$, and were hospitalized prior to intervention (51\%). Preoperatively, $28 \%$ had diabetes mellitus, $11 \%$ had a low ejection fraction $(\leq 40 \%)$, and $8 \%$ had renal insufficiency (creatinine $>176$ $\mathrm{mmol} / \mathrm{L}$ ). 
Table 1. Preoperative Clinical Characteristics

\begin{tabular}{|c|c|c|c|}
\hline \multirow{2}{*}{ Characteristics } & \multicolumn{2}{|c|}{ Infection cases } & \multirow{2}{*}{$P$ value } \\
\hline & Yes, $n=39(\%)$ & No, $n=146(\%)$ & \\
\hline Age (years) & & & 0.0185 \\
\hline$<60$ & $7(17.9)$ & $54(37.0)$ & \\
\hline $60-69$ & $12(30.8)$ & $51(34.9)$ & \\
\hline $70-79$ & $14(35.9)$ & $23(15.8)$ & \\
\hline$\geq 80$ & $6(15.4)$ & $18(12.3)$ & \\
\hline Female & $7(17.9)$ & $32(21.9)$ & $\mathrm{n} / \mathrm{s}$ \\
\hline $\mathrm{EF}<40 \%$ & $6(15.4)$ & $14(9.6)$ & $\mathrm{n} / \mathrm{s}$ \\
\hline PVD and/or CVD & $15(38.5)$ & $47(32.2)$ & $\mathrm{n} / \mathrm{s}$ \\
\hline Renal insufficiency $(\mathrm{Cr}>176)$ & $4(10.3)$ & $10(6.8)$ & $\mathrm{n} / \mathrm{s}$ \\
\hline Diabetes & $13(33.3)$ & $39(26.7)$ & $\mathrm{n} / \mathrm{s}$ \\
\hline $\mathrm{CHF}$ & $5(10.2)$ & $14(9.6)$ & $\mathrm{n} / \mathrm{s}$ \\
\hline COPD & $7(17.5)$ & $16(11.0)$ & $\mathrm{n} / \mathrm{s}$ \\
\hline Redo surg. & $1(2.6)$ & $11(7.5)$ & $\mathrm{n} / \mathrm{s}$ \\
\hline Procedure type & & & $\mathrm{n} / \mathrm{s}$ \\
\hline Isol. CABG & $20(51.3)$ & $76(52.1)$ & \\
\hline Isol. valve & $5(12.8)$ & $29(19.9)$ & \\
\hline CABG + valve & $6(15.4)$ & $10(6.8)$ & \\
\hline Complex procedure & $8(20.5)$ & $31(21.2)$ & \\
\hline Status & & & $\mathrm{n} / \mathrm{s}$ \\
\hline Elective & $14(35.9)$ & $76(52.1)$ & \\
\hline In-house & $22(56.4)$ & $60(41.1)$ & \\
\hline Urgent $(<4 \mathrm{~h})$ & $2(5.1)$ & $7(4.8)$ & \\
\hline Emergency & $1(2.6)$ & $3(2.1)$ & \\
\hline NYHA class & & & $\mathrm{n} / \mathrm{s}$ \\
\hline I & $1(2.9)$ & $15(12.3)$ & \\
\hline II & $11(32.4)$ & $39(32.0)$ & \\
\hline III & $19(55.9)$ & $51(41.9)$ & \\
\hline IV & $3(8.8)$ & $17(13.9)$ & \\
\hline
\end{tabular}

CABG: coronary artery bypass graft; CHF: congestive heart failure; COPD: chronic obstructive pulmonary disease; Cr: creatinine; CVD: cerebral vascular disease; EF: ejection fraction; Isol: isolated; NYHA: New York Heart Association; PVD: peripheral vascular disease; n/s: not significant.

A total of 39 patients (21\%) developed at least one HAI during their indexed cardiac surgical admission, defined from the time of the operation until discharge from hospital (Table 1). All infection definitions were based on established STS definitions and represent the standard of reporting in the cardiac surgical literature [3]. Unadjusted findings suggest that infection occurs more often in patients presenting with the following clinical conditions: older age, male sex, low ejection fraction, peripheral vascular disease or cerebral vascular disease, renal insufficiency, diabetes mellitus, congestive heart failure, chronic obstructive pulmonary disease (COPD), and complex procedures other than isolated CABG. The most common infection was UTIs ( $8 \%$ ), followed by pneumonia (7\%), leg harvest site infection (5\%), and sSSI (4\%). Sepsis was the least common infection and was only seen in $2 \%$ of patients (Table 2 ). There were no cases of dSSI in the period examined. A total of six patients developed more than one type of infection during their hospital admission.

The overall in-house mortality rate for the entire study population was $3.8 \%$. However, the in-house mortality of patients with an HAI was $10 \%$, being much higher than patients without an infection, which was $2 \%(\mathrm{P}=0.0369)$. The median LOS for the entire population was 8 days. Similarly the median LOS for patients with an HAI was 14 days compared to 8 days for those who did not contract an infection $(\mathrm{P} \leq 0.0001)$. This study used a standard definition of LOS exceeding 9 days as prolonged LOS, consistent with our previous study [3]. In patients that contracted an HAI, $72 \%$ had an LOS exceeding 
Table 2. Unadjusted Postoperative Outcomes

\begin{tabular}{|c|c|c|c|}
\hline \multirow{2}{*}{ Outcome } & \multicolumn{2}{|c|}{ Infection cases } & \multirow{2}{*}{ P value } \\
\hline & Yes, $n=39(\%)$ & No, $n=146(\%)$ & \\
\hline Mortality & $4(10.3)$ & $3(2.1)$ & 0.0369 \\
\hline LOS $>9$ days & $28(71.8)$ & $50(34.2)$ & $<0.0001$ \\
\hline \multicolumn{4}{|l|}{ Infection type } \\
\hline UTI & $14(35.9)$ & - & \\
\hline Pneumonia & $12(30.8)$ & - & \\
\hline Leg harvest site & $9(23.1)$ & - & \\
\hline sSSI & $8(20.5)$ & - & \\
\hline Sepsis & $3(7.7)$ & - & \\
\hline
\end{tabular}

LOS: length of stay; sSSI: superficial surgical site infection; UTI: urinary tract infection.

9 days versus only $34 \%$ of non-infected patients $(\mathrm{P} \leq 0.0001)$.

\section{Variation in clinical practice with regard to HAIs}

All individual patient records were reviewed to determine the circumstances surrounding the diagnosis of an infection. Specifically, confirmation with bacteriology differed significantly across infection types: $100 \%$ in patients diagnosed with sepsis, $67 \%$ in patients with UTIs and pneumonia, $25 \%$ in sSSI, and only $10 \%$ in patients with a leg infection (Fig. 1). The average time for the diagnosis of infection postoperatively was $6.3 \pm 4.0$ days (Fig. 2). There was no significant difference in the detection times between infection types analyzed, ranging from 5.8 days for leg infections to 7.2 days for pneumonia. A large diversity of bacterial species was detected in the period studied and varied based on infection type. Specific bacteria associated with each type of infection were identified (Table 3). To the best of our knowledge, there was no case of methicillin-resistant Staphylococcus aureus (MRSA) or vancomycin-resistant enterococcus (VRE) dur- ing the study period.

The range of treatment duration was from 4 to 16 days. However, overall antimicrobial treatment duration was consistent across infection types with the exception of superficial surgical site infections, which had median treatment duration that was 3 days greater than all other infections being investigated (Fig. 3). This was in spite of 16/39 (41\%) infections not confirmed by a positive culture. All patients with unverified HAIs did not have antimicrobials discontinued after negative cultures were obtained.

\section{Discussion}

The present study represents a 2-month detailed review of all reported infection occurring in all cardiac surgery performed at the only cardiac center for the Province of Nova Scotia, Canada. In our study period, the overall rate of HAIs was $21 \%$ $(39 / 185)$, similar to recently published data suggesting that HAI prevalence in cardiac surgery patients was $20 \%$ in the last 5 years and comparable to similar patient populations $[3,11$,

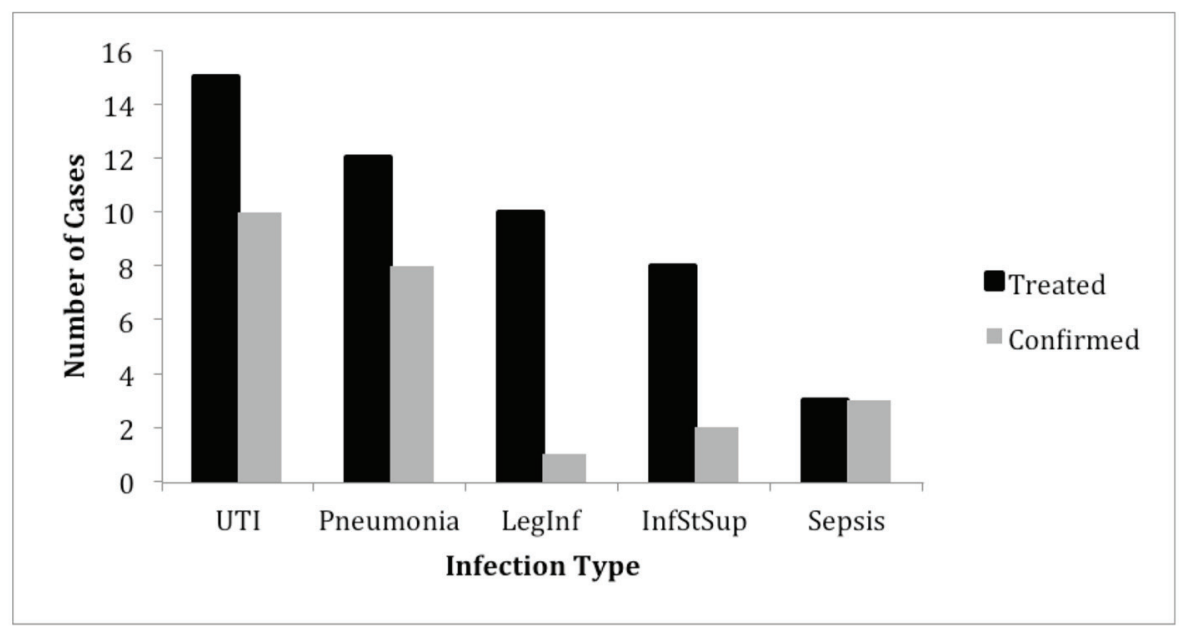

Figure 1. Total number of infections treated vs. confirmed with positive lab cultures. UTI: urinary tract infection; LegInf: leg infection; InfStSup: superficial surgical site infection. 


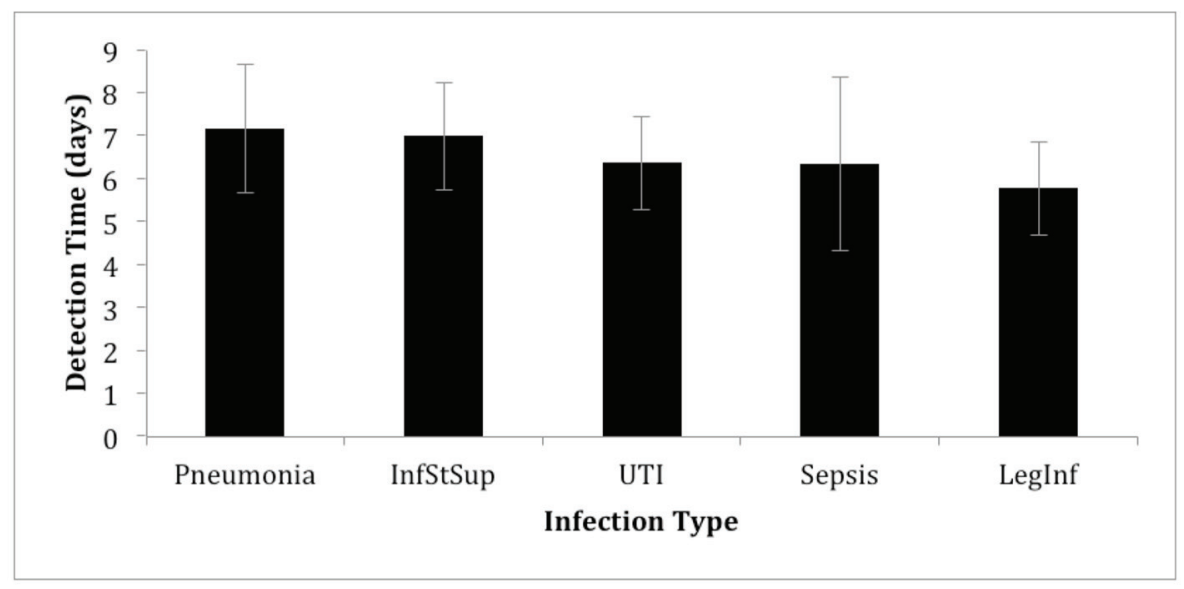

Figure 2. Mean time until infection was detected starting from the time of surgery. InfStSup: superficial surgical site infection; UTI: urinary tract infection; Leglnf: leg infection.

12]. The present study was not designed to focus on risk factors for HAI as previously published [3], but instead prompted the present review of current clinical practice in the setting of suspected infection. We specifically looked at how commonly suspected HAIs were treated, verified through microbiology tests and in the case of unconfirmed HAIs, if antimicrobial treatment was discontinued.

We found that a significant number $(38 \%)$ of suspected infections were not verified or confirmed by positive microbiology cultures. Furthermore, our findings demonstrate that

Table 3. Verified Microbiology Cultures

\begin{tabular}{lll}
\hline Infection type & Organism & Cases \\
\hline UTI & Escherichia coli & 7 \\
& Pseudomomas aeruginosa & 2 \\
& Citrobacter youngae & 1 \\
& Serratia marcescens & 1 \\
& Candida albicans & 1 \\
& Klebsiella pneumoniae & 1 \\
\hline \multirow{4}{*}{ Pneumonia } & Morganella morganii & 1 \\
& Haemophilus influenzae & 3 \\
& Pseudomonas aeruginosa & 2 \\
& Escherichia coli & 1 \\
& Serratia marcescens & 1 \\
& Staphylococcus aureus & 1 \\
\hline Sepsis & Streptococcus pneumoniae & 1 \\
& Proteus vulgaris & 1 \\
\hline & Enterobacter cloacae & 1 \\
\hline & Cellulitis*** & 1 \\
& Coagulase-negative staphlococci & 2 \\
& Candida albicans & 2 \\
& Coagulase-negative staphlococci & 1 \\
\hline
\end{tabular}

sSSI: superficial surgical site infection; UTI: urinary tract infection. in all patients in which negative cultures were obtained (16 out of 39), none of these patients had antimicrobial treatment discontinued. This is not surprising for infections such as sSSI where bacterial confirmation may not be of much clinical value. However, our findings do show that clinicians appear to generally continue a course of antimicrobials even in the context of clinical improvement and lack of correlating evidence such as bacteriology. Evidence supporting antimicrobial stewardship by treating clinicians has been limited in cardiac surgery but shown in other areas to be safe and appropriate [5, $10,13]$. Effective antimicrobial stewardship relies on selecting the best drug at an optimal dosage and duration to effectively treat an infection while simultaneously reducing the selection of antimicrobial resistant bacteria [11]. Numerous studies have shown that antimicrobial use can lead to selective pressure for resistance and other side effects [14-16]. One should note, however, that there were no cases of antimicrobial resistant bacteria in the period studied, such as MRSA or VRE and these low numbers are consistent with what we have published before [3].

Our findings highlight physician's tendency to continue treatment with antimicrobials in times where it has been demonstrated in select cases to safely discontinue these drugs and limit antimicrobial use $[15,17]$. Our findings are in keeping with similar reports looking at nosocomial pneumonia where it was shown that once antimicrobials were prescribed, it was continued in all patients, including those with negative cultures [6]. Although the effects of improperly prescribing antimicrobials are commonly known among physicians, translating knowledge into policies that are adequate and effectively implemented still remains a significant problem [15]. There are many published guidelines on the implementation of effective antimicrobial stewardship, which involves streamlining or de-escalating therapy once cultures are received. It is believed that by doing so, the causative pathogen can be more appropriately treated, selective pressure for antimicrobial resistant bacteria is decreased, and cost savings can be achieved [13]. We show here that while these guidelines are well known, their translation into clinical practice may not be optimal and 


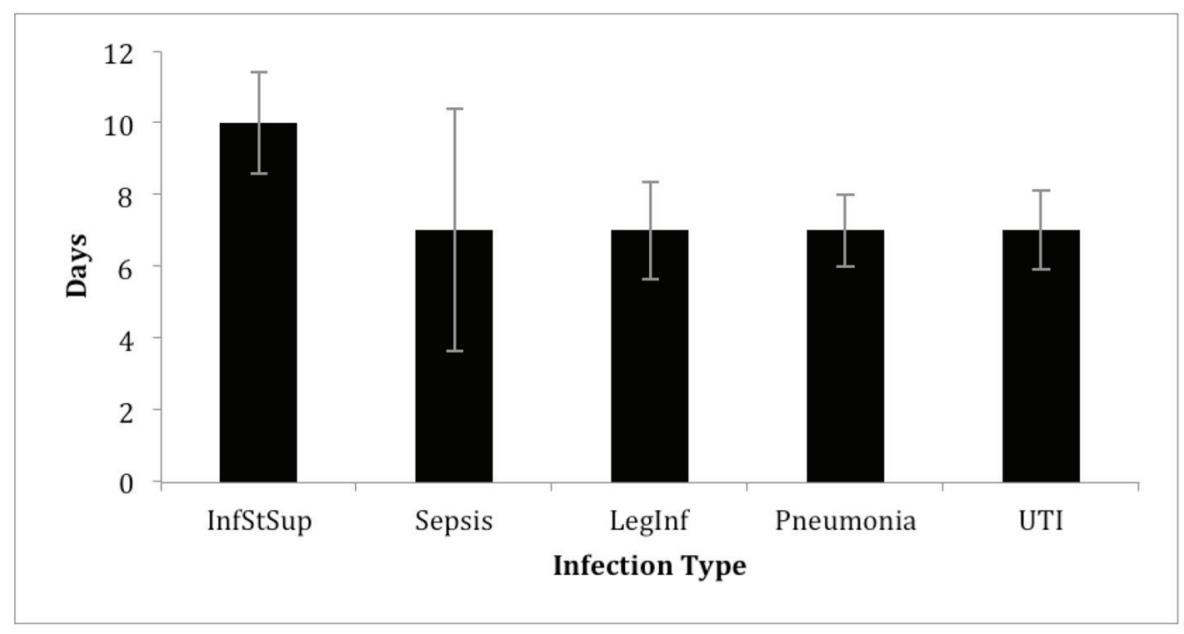

Figure 3. Median duration of antimicrobial treatment. InfStSup: superficial surgical site infection; LegInf: leg infection; UTI: urinary tract infection.

require more concerted effort.

We have shown with this study the prevalence of different types of infections in postoperative cardiac surgery patients. Consistent with previous studies, UTIs and pneumonia are the most common types of infections postoperatively while sepsis occurs in a much smaller segment of patients $[1,3]$. The overall rate of infection was within the range of other studies investigating HAIs, which ranged from $7 \%$ to $20 \%[11,12]$. The time until detection of infection was consistent across the different types identified, averaging 6.3 days. We believe the narrow range of detection time across infections may be due to similarities in the patient population such as comorbidities and types of surgical procedures performed and reflect a clean type of procedure in which infection should be rare in the first few days after surgery. Treatment duration was also consistent across infections allowing a $7-10$ day course of antibiotics with the exception of surgical site infections, which had a median duration of 3 days greater than all other infections. We speculate this may be due to the significant concern physicians have regarding surgical site infections in patients requiring a sternotomy to avoid a deep sternal wound complication which is known to be associated with significant morbidity and mortality.

While the sample size of the present study was relatively small, consisting of 185 patients, our findings were consistent with a recently published study from our group that looked at nearly 20,000 patients undergoing cardiac surgery. What is unique about our study is that it contains very detailed patient information obtained from direct chart review rather than registry data only. This included information on pathogens, antimicrobials administered, duration of treatment, and diagnostic criteria used. We acknowledge that examining infection at a single institution cannot address infections specific to all other regions, limiting the generalizability of our findings. However, the present study is from a large regional center responsible for all advanced cardiac care for a Province in Canada. Furthermore, the QEII HSC has received full national accreditation including implementation of national standards regarding
HAI prevention, making the conclusions relevant for Canadian health care providers.

\section{Conclusions}

Despite these limitations, our study was novel in several ways. Our study has shown that HAIs are common in modern cardiac care. Furthermore, we have shown that common types of infection are generally approached in a similar fashion by the clinician. This means that when suspected infections are treated clinicians prescribe a full course of antimicrobials without discontinuation even after negative lab results are received. While this study was not designed to review the application of published guidelines for antimicrobial stewardship, it does suggest some difficulty in translating this knowledge to clinical practice.

\section{Acknowledgments}

The authors thankfully acknowledge the Maritime Heart Center for providing access to the MHC registry and financial support for the present study from Nova Scotia Health Authority "Transitioning Research Into Care (TRIC)" program.

\section{Abbreviations}

HAI: hospital acquired infection; SIRS: systemic inflammatory response syndrome; QEII HSC: Queen Elizabeth II Health Sciences Center; MHC: Maritime Heart Center; HPF: horizon patient folder; STS: Society of Thoracic Surgeons; UTI: urinary tract infection; sSSI: superficial sternal site infection; dSSI: deep sternal site infection; LOS: length of stay; CABG: coronary artery bypass graft; COPD: chronic obstructive pulmonary disease; MRSA: methicillin-resistant Staphylococcus aureus; VRE: vancomycin-resistant enterococcus 


\section{References}

1. Kollef MH, Sharpless L, Vlasnik J, Pasque C, Murphy D, Fraser VJ. The impact of nosocomial infections on patient outcomes following cardiac surgery. Chest. 1997;112(3):666-675.

2. Brown PP, Kugelmass AD, Cohen DJ, Reynolds MR, Culler SD, Dee AD, Simon AW. The frequency and cost of complications associated with coronary artery bypass grafting surgery: results from the United States Medicare program. Ann Thorac Surg. 2008;85(6):1980-1986.

3. Mocanu V, Buth KJ, Johnston LB, Davis I, Hirsch GM, Legare JF. The Importance of Continued Quality Improvement Efforts in Monitoring Hospital-Acquired Infection Rates: A Cardiac Surgery Experience. Ann Thorac Surg. 2015;99(6):2061-2069.

4. van der Velden LB, Tromp M, Bleeker-Rovers CP, Hulscher M, Kullberg BJ, Mouton JW, Sturm PD, et al. Nonadherence to antimicrobial treatment guidelines results in more broad-spectrum but not more appropriate therapy. Eur J Clin Microbiol Infect Dis. 2012;31(7):1561-1568.

5. Dellit TH, Owens RC, McGowan JE, Jr., Gerding DN, Weinstein RA, Burke JP, Huskins WC, et al. Infectious Diseases Society of America and the Society for Healthcare Epidemiology of America guidelines for developing an institutional program to enhance antimicrobial stewardship. Clin Infect Dis. 2007;44(2):159-177.

6. Singh N, Rogers P, Atwood CW, Wagener MM, Yu VL. Short-course empiric antibiotic therapy for patients with pulmonary infiltrates in the intensive care unit. A proposed solution for indiscriminate antibiotic prescription. Am J Respir Crit Care Med. 2000;162(2 Pt 1):505-511.

7. Paterson DL, Rice LB. Empirical antibiotic choice for the seriously ill patient: are minimization of selection of resistant organisms and maximization of individual outcome mutually exclusive? Clin Infect Dis. 2003;36(8):10061012.

8. Michalopoulos A, Geroulanos S, Rosmarakis ES, Falagas ME. Frequency, characteristics, and predictors of micro- biologically documented nosocomial infections after cardiac surgery. Eur J Cardiothorac Surg. 2006;29(4):456460.

9. Darby JM, Linden P, Pasculle W, Saul M. Utilization and diagnostic yield of blood cultures in a surgical intensive care unit. Crit Care Med. 1997;25(6):989-994.

10. Kaki R, Elligsen M, Walker S, Simor A, Palmay L, Daneman N. Impact of antimicrobial stewardship in critical care: a systematic review. J Antimicrob Chemother. 2011;66(6):1223-1230.

11. Rahmanian PB, Kroner A, Langebartels G, Ozel O, Wippermann J, Wahlers T. Impact of major non-cardiac complications on outcome following cardiac surgery procedures: logistic regression analysis in a very recent patient cohort. Interact Cardiovasc Thorac Surg. 2013;17(2):319326; discussion 326-317.

12. Alasmari FA, Tleyjeh IM, Riaz M, Greason KL, Berbari EF, Virk A, Baddour LM. Temporal trends in the incidence of surgical site infections in patients undergoing coronary artery bypass graft surgery: a population-based cohort study, 1993 to 2008. Mayo Clin Proc. 2012;87(11):10541061.

13. Fishman N. Antimicrobial stewardship. Am J Med. 2006;119:53-61.

14. Fuller JD, Low DE. A review of Streptococcus pneumoniae infection treatment failures associated with fluoroquinolone resistance. Clin Infect Dis. 2005;41(1):118121.

15. Marshall DA, McGeer A, Gough J, Grootendorst P, Buitendyk M, Simonyi S, Green K, et al. Impact of antibiotic administrative restrictions on trends in antibiotic resistance. Can J Public Health. 2006;97(2):126-131.

16. Vanderkooi OG, Low DE, Green K, Powis JE, McGeer A. Predicting antimicrobial resistance in invasive pneumococcal infections. Clin Infect Dis. 2005;40(9):1288-1297.

17. Sunenshine RH, Liedtke LA, Jernigan DB, Strausbaugh LJ. Role of infectious diseases consultants in management of antimicrobial use in hospitals. Clin Infect Dis. 2004;38(7):934-938. 